\title{
Weight-Based, Low-Dose Pediatric Whole-Body PET/CT Protocols
}

Adam M. Alessio ${ }^{1,2}$, Paul E. Kinahan ${ }^{1}$, Vivek Manchanda ${ }^{1,2}$, Victor Ghioni ${ }^{2}$, Lisa Aldape ${ }^{2}$, and Marguerite T. Parisi ${ }^{1,2}$

${ }^{1}$ Department of Radiology, University of Washington, Seattle, Washington; and ${ }^{2}$ Department of Radiology, Seattle Children's Hospital, Seattle, Washington

Adult PET/CT acquisition protocols need to be modified for pediatric imaging to minimize the radiation dose while maintaining diagnostic utility. We developed pediatric PET/CT acquisition protocols customized to patient weight and estimated the dosimetry and cancer risk of these low-dose protocols to communicate basic imaging risks. Methods: Protocols were developed for whole-body ${ }^{18} \mathrm{~F}-\mathrm{FDG}$ imaging of patients in PET mode with a weight-based injected activity (5.3 $\mathrm{MBq} / \mathrm{kg}$ ) and acquisition times (3-5 min/field of view) and for CT for attenuation correction and localization with a weightbased tube current ranging from 10 to $40 \mathrm{mAs}$. Patients were categorized on the basis of the Broselow-Luten color-coded weight scale. Dosimetry and radiation-induced cancer risk for the PET and CT acquisition in each category were derived from mean patient sizes and the interpolation of factors from accepted patient models. Results: Whole-body pediatric PET/CT protocols require the customization of PET-acquisition settings and task-specific selection of CT technique. The proposed weight-based protocols result in an approximate effective dose ranging from $8.0 \mathrm{mSv}$ for a 9-kg patient up to $13.5 \mathrm{mSv}$ for a 63-kg patient. The radiation dose from the proposed protocols is $20 \%-50 \%$ (depending on patient weight), the dose from PET/ CT protocols that use a fixed CT technique of $120 \mathrm{mAs}$ and $120 \mathrm{kVp}$. The approximate, conservative estimate of additional lifetime attributable risk (LAR) of cancer incidence for females using the proposed protocols was approximately 3 in 1,000, with a variation of $18 \%$ across patient categories. For males, the additional LAR of cancer incidence was approximately 2 in 1,000, with a variation of $16 \%$ across categories. Conclusion: Lowdose PET/CT protocols for 11 patient weight categories were developed. The proposed protocols offer an initial set of acquisition parameters for pediatric PET/CT. The use of multiple categories allows for the continued refinement of dose-reduction parameters to minimize dose while maintaining image quality across the range of pediatric patient sizes.

Key Words: pediatric PET/CT; dosimetry; low-dose CT; acquisition protocols

J Nucl Med 2009; 50:1570-1578

DOI: 10.2967/jnumed.109.065912

Received May 7, 2009; revision accepted Jul. 10, 2009.

For correspondence or reprints contact: Adam M. Alessio, Department of Radiology, University of Washington, P.O. Box 357987, Old Fisheries

Center, Room 220, 4000 15th Ave., NE, Seattle, WA 98195-7987.

E- mail: aalessio@u.washington.edu

COPYRIGHT $\odot 2009$ by the Society of Nuclear Medicine, Inc.
$\mathbf{T}$ he combination of PET and CT is a well-established tool for adult medical imaging and has proven value for oncology, cardiology, and neurology. As the availability of these dualmodality systems increases, PET/CT is of growing importance in pediatric imaging - particularly for cancer detection, staging, therapeutic response monitoring, and outcome prediction (1). Adult PET/CT protocols should be appropriately modified for application to the pediatric population. Several works have offered recommendations on pediatric PET/CT protocols (2-4). In general, pediatric protocols are similar to adult protocols. Some variations that may require special attention include ensuring that the patient is quiescent during the uptake phase, providing a warm environment during the uptake phase, and determining whether sedation and imaging of the extremities is warranted. Development of pediatric protocols also requires the careful selection of PET and CT acquisition parameters to minimize the dose while maintaining task-dependent image quality. A particular concern is the radiation exposure from the PET and CT portions of the examination in this younger and more radiation-sensitive population. The radiation-induced risk of adverse health effects is greater in children than in adults. For context, analyses of atomic bomb survivors suggest that the lifetime risk of cancer for those exposed to radiation at age 5 is 1.7-2.1 times greater than for those exposed at age 20 (5). This increased risk has received recent attention in the imaging community, leading to numerous efforts to minimize pediatric dose, particularly for CT $(6,7)$.

This work presents pediatric PET/CT protocols tailored for multiple patient categories. The proposed protocols are intended for patients referred for a PET/CT procedure who do not require stand-alone diagnostic CT or contrast-enhanced CT. The intent is to outline a set of whole-body PET/CT acquisition parameters that, to some extent, optimize the tradeoff between image quality and risk from radiation dose. We acknowledge that both sides of this tradeoff are difficult to quantify. On the one side, the image quality of dualmodality, volumetric data is task-dependent and therefore challenging to measure. For this scan population, we need PET/CT images that can complete multiple tasks: detect, stage, and monitor diverse disease. Evaluating performance of these multiple tasks is complicated to impossible. 
The other side of the tradeoff, risk from radiation, is even more challenging to establish. First, it is difficult to accurately estimate radiation dosimetry from CT and PET procedures in the diverse, pediatric population with varying morphology, organ sizes, and tissue kinetics. Moreover, we have extremely limited knowledge of the long-term risk from these low levels of radiation.

Accepting these limitations in quantifying both image quality and risk, we proposed an initial set of whole-body pediatric PET/CT protocols. This set is derived from our experience of acceptable image quality and current, conservative estimates of radiation risk from PET/CT acquisitions. The goal was to offer a set of protocols that could be refined by practitioners to meet their standards for image quality and to provide risk information for whole-body PET/CT examinations for communication with referring physicians. This work provides preliminary results that lay the groundwork for discussions of risk versus benefit of pediatric PET/CT procedures. A thorough risk-versus-benefit discussion of procedures would need to incorporate other risks or benefits associated with diagnostics, including treatment changes, therapy choices, and outcomes, but is beyond the scope of this project.

\section{MATERIALS AND METHODS}

\section{PET/CT Protocol Design}

We developed weight-based, whole-body ${ }^{18} \mathrm{~F}-\mathrm{FDG}$ PET/CT protocols and estimated the radiation exposure and cancer risk of these examinations. All patients underwent identical preparation (6-h nothing by mouth except water, warm and quiescent uptake environment), were scanned $1 \mathrm{~h}$ after injection of ${ }^{18} \mathrm{~F}-\mathrm{FDG}$, and were imaged during quiet breathing (non-breath hold) for the PET and $\mathrm{CT}$ acquisition. The PET portion preceded the CT portion to reduce the potential for respiratory and other motion mismatch and, in the case of contrast-enhanced CT examinations, reduce the potentially uncomfortable scan time after injection of CT contrast. Shorter patients (height, $<120 \mathrm{~cm}$ ) were scanned from head to toe; taller patients or those with pelvic pathology were scanned bladder up. All images were acquired on a 64-slice PET/CT scanner (Discovery VCT [DVCT]; GE Healthcare) (8).
The pediatric population was divided into 11 weight-based categories based on the Broselow-Luten color scale. This scale was originally devised for emergency pediatrics (9) and also used by Frush et al. for pediatric CT protocols (10).

PET Technique. For each category, we customized both PET and CT acquisition settings, as shown in Table 1. For the PET examination, we varied the injected ${ }^{18} \mathrm{~F}-\mathrm{FDG}$ activity $(5.3 \mathrm{MBq} /$ $\mathrm{kg} ; 37 \mathrm{MBq}$ minimum/370 $\mathrm{MBq}$ maximum) and acquisition times (3-5 min/field of view [FOV]). All patients were scanned in 3-dimensional mode, and images were reconstructed with 3dimensional ordered-subset expectation maximization (3 iterations, 28 subsets, 7-mm postsmoothing). The PET system had a 15.7-cm axial FOV; with bed overlap, the effective FOV was 12.6 $\mathrm{cm}$, leading to 5-12 scanning bed positions, depending on patient height.

CT Technique. The CT technique was designed for attenuation correction and anatomic correlation of the PET findings. Patients referred for diagnostic CT procedures should not be imaged with the following low-dose protocol. All patients were scanned with a tube voltage of $120 \mathrm{kVp}$, pitch of $0.98: 1$, rotation speed of $0.5-\mathrm{s}$, body bow-tie filter, and reconstructed slice thickness of $2.5 \mathrm{~mm}$, and we varied the maximum CT tube current (10-40 mAs) for each category. In our clinical practice, we initially used a slightly higher CT technique and gradually decreased the technique over several months to a level accepted by all radiologists in our clinic. Although this is not a rigorous justification, visual consensus of CT technique is a primary approach that institutions use to determine their CT acquisition settings (11).

To further support the selected CT protocols, we scanned 4 water-filled cylinders of varying circumferences $(33,40,64$, and $79 \mathrm{~cm}$ ) with a range of CT techniques. To estimate dosimetry in average-sized patients, these cylinders were related to anthropomorphic phantoms of newborns and 4-mo-, 8-y-, and 14-y-olds according to the relationship presented by Huda et al. (12). The dose to each phantom was estimated as discussed in the "Dosimetry Estimation" section. We scanned each cylinder with the same basic settings used for CT (pitch, 0.98:1; rotation, $0.5 \mathrm{~s}$; slice thickness, $2.5 \mathrm{~mm}$ ). Images were acquired with tube voltages of $80,100,120$, and $140 \mathrm{kVp}$ and tube currents of $10,20,40$, and 80 mAs. Noise in the images was determined from the SD in 15 circular regions from 3 adjacent slices.

If anatomic correlation of the PET image is not necessary, lower tube voltages and currents can be used without degradation

\section{TABLE 1. Pediatric Whole-Body PET/CT Protocols Highlighting Acquisition Settings Varied for Each Weight Category}

\begin{tabular}{|c|c|c|c|c|c|}
\hline \multicolumn{3}{|c|}{ Patient } & \multirow{3}{*}{$\begin{array}{l}\text { CT acquisition, } \\
\text { maximum tube } \\
\text { current for CT (mAs) }\end{array}$} & \multirow{2}{*}{\multicolumn{2}{|c|}{ PET acquisition }} \\
\hline & & Estimated & & & \\
\hline Category & $\begin{array}{l}\text { Weight range } \\
(\mathrm{kg})\end{array}$ & $\begin{array}{c}\text { whole-body } \\
\text { scan range }(\mathrm{mm})\end{array}$ & & $\begin{array}{l}\text { Injected activity } \\
\text { (MBq [mCi]) }\end{array}$ & $\begin{array}{l}\text { Scan time per } \\
\text { FOV (min) }\end{array}$ \\
\hline Pink & $6-7.4$ & $59.5-66.5$ & 10 & $37(1.0)$ & 3 \\
\hline Red & $7.5-9.4$ & $66.5-74$ & 10 & 45 (1.2) & 3 \\
\hline Purple & $9.5-11.4$ & $74-84.5$ & 15 & 55 (1.5) & 3 \\
\hline Yellow & $11.5-14.4$ & $84.5-97.5$ & 20 & 68 (1.9) & 3 \\
\hline White & $14.5-18.4$ & $97.5-110$ & 20 & $87(2.4)$ & 3 \\
\hline Blue & $18.5-22.4$ & $110-122$ & 20 & 108 (2.9) & 3 \\
\hline Orange & $22.5-31.4$ & $122-137$ & 25 & $142(3.9)$ & 5 \\
\hline Green & $31.5-40.5$ & $137-150$ & 30 & $190(5.1)$ & 5 \\
\hline Black & $40.5-55$ & $150-162$ & 30 & $252(6.8)$ & 5 \\
\hline Small adult & $55-70$ & $162-170$ & 35 & 330 (8.9) & 5 \\
\hline Large adult & $>70$ & $>170$ & 40 & $370(10.0)$ & 5 \\
\hline
\end{tabular}


of the attenuation-corrected PET images (13). For a discussion of the tradeoffs and motivations for different CT acquisitions in pediatric PET/CT, readers are referred to the work by Gelfand and Lemen (14).

\section{Dosimetry Estimation}

The dosimetry from radiologic examinations has been estimated from both measured studies and Monte Carlo simulations (15-17). In general, these estimates are acknowledged as rough approximations of radiation dose and are appropriate when used to compare procedures and to develop a populationwide concept of the dose. All of these approaches are imperfect when applied to specific patients because they were derived from a small set of standard models of patient morphology, biokinetics, and organ sizes.

For this study, we estimate effective dose, which accounts for the dose to irradiated organs and the relative radiosensitivity of each organ. The effective dose is the accepted dose metric for quantifying the stochastic risk of carcinogenic or genetic effects and is an effective method to compare relative radiation dose from different imaging techniques (18). The effective dose is the sum of the equivalent dose to each organ multiplied by the radiosensitive weighting factor for that organ. We used organ weighting from the International Commission on Radiological Protection (ICRP) publication 60 (19). Radiation in the form of annihilation and $\mathrm{x}$ ray photons from PET and CT is considered low-linear energy transfer (LET) because it causes ionizations sparsely throughout a cell and is less destructive than radiation from subatomic particles. For low-LET radiation, the radiation weighting factor is 1 , so the equivalent dose (in sieverts or rems) is equal to the absorbed dose (in grays or rads). Therefore, in this work $1 \mathrm{~Sv}$ equals $1 \mathrm{~Gy}$ for individual tissues. The units of the combined whole-body effective dose are always sieverts or rems $(1 \mathrm{~Sv}=100 \mathrm{rem})$.

We report the effective dose and the background equivalent radiation time (BERT), the time in years to obtain the same effective dose from background. The BERT is calculated by dividing the effective dose by the average natural background radiation $(3 \mathrm{mSv} / \mathrm{y})$ received from living in the United States $(20)$.

In this work, we used simulation-derived dosimetry estimates and interpolate the estimates to our specific categories based on weight for PET and on age for CT. The age for each category was determined from standard growth charts (21), calculated as the average of the 50th-percentile male and female age for each weight category.
PET Dosimetry. For the PET dosimetry, estimates were derived from factors in ICRP 80 (17). The ICRP 80 values are based on simulations of 1-, 5-, 10-, and 15-y-olds and adult models. ICRP simulations assume normal renal function and an age-appropriate urinary bladder emptying rate.

The ICRP 80 does not include dose estimates for a newborn model. A primary challenge of radionuclide dosimetry estimation for infants is that the biokinetics of the tracer can vary substantially between individuals of the same age. We used estimates from a biokinetic model averaged across 10 low-birth-weight newborns prepared by Niven and Nahmias (22). The effective dose factors per activity injected are presented in Table 2. The dose for each patient category was estimated by linear interpolation of the factors based on the average weight in each category.

CT Dosimetry. Dosimetry was estimated for the CT acquisition using simulated dose computations of an adult from the U.K. National Radiological Protection Board (16) and the ImPACT CT Dosimetry Calculator (23). The effective dose from the adult model was interpolated to each pediatric category based on scaling factors from Khursheed et al. (24). The scaling factors for the scan of the trunk with a GE Healthcare 9800 scanner are presented in Table 2 and are in close agreement with measured results from Huda and Ogden (25). We used the reported 9800 scanner factors to best match our current GE Healthcare Discovery VCT PET/CT system, which also uses a bow-tie shaping filter, leading to proportionally greater factors than less-filtering systems for smaller patients. The effective dose for each of our patient categories was determined by linear interpolation of the pediatric scaling factors based on the average age in each category.

\section{Cancer Risk Estimation}

The National Academies of Sciences Biologic Effects of Ionizing Radiation (BEIR) VII phase 2 report synthesized numerous epidemiologic data sources to offer a connection between health risks and radiation dose (5). The quantitative risk estimates in the BEIR VII report are based on studies of atomic bomb survivors in Japan and are in keeping with results from analyses of radiation safety workers (26). These estimates are predicated on the linear no-threshold model of the relationship between lowdose exposures to radiation and cancer. This model assumes that the risk of solid cancer incidence at low levels can be extrapolated from linear fits to the risk of cancer incidence at high levels. Although this remains an active area of debate $(27,28)$, several sources have argued that this is a prudent working assumption

\begin{tabular}{|c|c|c|c|c|c|c|}
\hline \multirow[b]{2}{*}{ Procedure } & \multicolumn{6}{|c|}{ Relative dose factor by age } \\
\hline & Adult & $15 y$ & $10 y$ & $5 y$ & $1 \mathrm{y}$ & Newborn \\
\hline \multicolumn{7}{|l|}{ PET (17) } \\
\hline $\mathrm{mSv} / \mathrm{mCi}$ & 0.70 & 0.93 & 1.3 & 1.9 & 3.5 & $7.8^{\star}$ \\
\hline $\mathrm{mSv} / \mathrm{MBq}$ & $1.9 \times 10^{-2}$ & $2.5 \times 10^{-2}$ & $3.6 \times 10^{-2}$ & $5.0 \times 10^{-2}$ & $9.5 \times 10^{-2}$ & $2.1 \times 10^{-1 *}$ \\
\hline Nominal weight $(\mathrm{kg})$ & 70 & 58 & 32 & 19 & 9.8 & $1.0^{*}$ \\
\hline $\begin{array}{l}\text { CT (relative to adult } \\
\text { effective dose) (24) }\end{array}$ & 1 & 1.15 & 1.42 & 1.62 & 1.85 & 2.25 \\
\hline
\end{tabular}


TABLE 3. Additional LAR of All Forms of Cancer from

$100 \mathrm{mSv}$ of Low-LET Radiation

\begin{tabular}{|c|c|c|c|c|}
\hline \multirow{2}{*}{$\begin{array}{l}\text { Age at } \\
\text { exposure }\end{array}$} & \multicolumn{2}{|c|}{ Cancer incidence } & \multicolumn{2}{|c|}{ Cancer mortality } \\
\hline & Males (\%) & Females (\%) & Males (\%) & Females (\%) \\
\hline Newborn & 2.56 & 4.78 & 1.10 & 1.77 \\
\hline $5 y$ & 1.82 & 3.38 & 0.85 & 1.35 \\
\hline $10 y$ & 1.45 & 2.61 & 0.71 & 1.10 \\
\hline $15 y$ & 1.18 & 2.06 & 0.60 & 0.91 \\
\hline $20 y$ & 0.98 & 1.65 & 0.51 & 0.76 \\
\hline Baseline LAR & 45.5 & 36.9 & 22.1 & 17.5 \\
\hline
\end{tabular}

given the current, limited knowledge of the risks associated with low levels of radiation (29).

The risk of cancer incidence is a function of effective dose, sex, and age. In general, females are more susceptible to radiationinduced cancer than males. As expected, younger patients are at greater risk than older patients, because they are more radiosensitive and have more remaining years of life for a radiationinduced cancer to develop. In this work, we estimated the combined risk of all cancers including all solid cancers and leukemia. We linearly interpolated the BEIR VII risk estimates at fixed ages to the average ages in each of our patient categories. Table 3 lists the estimates from the BEIR VII report in terms of additional lifetime attributable risk (LAR) from $100 \mathrm{mSv}$ of radiation. These risks are in addition to the baseline LAR of cancer from all causes. For example, if a newborn boy receives $100 \mathrm{mSv}$ of radiation, the probability of cancer incidence in his lifetime will increase $2.6 \%$ from $45.5 \%$ to $48.1 \%$.

\section{RESULTS}

\section{PET Technique}

Figure 1 plots the proposed injected PET activity based on the linear scaling with weight and the recommendations from the European Association of Nuclear Medicine (EANM) pediatric dose card (30).

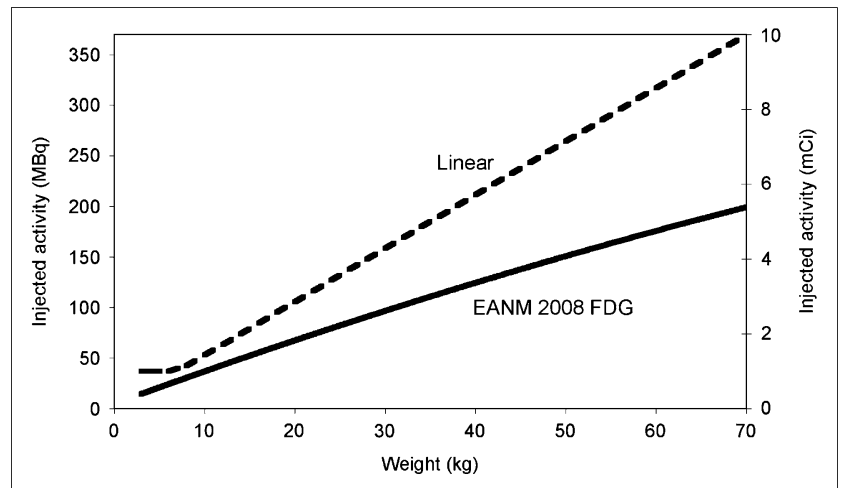

FIGURE 1. Injected ${ }^{18}$ F-FDG dose vs. weight for proposed linear approach and EANM pediatric guidelines.

\section{CT Technique}

The CT phantom acquisitions resulted in estimates of image noise in water phantoms for a range of tube voltages and currents; a portion of these noise measurements appears in Figure 2. As expected, noise decreases for increasing voltage, and for every quadrupling of tube current the noise decreases by a factor of 2 . Using this tube current-tonoise relationship, we can calculate the appropriate current level to achieve a fixed noise level. Clinical experience from patient examinations guided us to fix 17.6 Hounsfield units as the acceptable noise level for the $64-\mathrm{cm}$ phantom, as marked with an $\mathrm{X}$ in Figure 2B. To achieve this noise level, we can determine the CT technique for each voltage level for each phantom as presented in Figure 3. Then, with this voltage, current, and effective patient age (12), we can estimate the effective dose to achieve this noise level. Figure 4 presents the effective dose for a fixed noise level and offers insight into the optimal voltage to minimize dose. Results suggest that if small patients $(<1$ y) are scanned with $100 \mathrm{kVp}$, we could reduce the dose by approximately $7 \%$ and achieve the same noise level. Also, the optimal voltage for larger patients could be $140 \mathrm{kVp}$, leading to an approximately $15 \%$ dose reduction.

At present, we have used the proposed acquisition protocols on 140 patients across the range of patient categories. Qualitative review of these examinations by 2 nuclear medicine-trained radiologists revealed that all CT acquisitions were acceptable to localize the PET activity to anatomy. For example, Figure 5 presents a 6-kg "pink" patient scanned with $10 \mathrm{mAs}$. Despite the low-dose acquisition, the CT image has acceptable contrast and noise levels.

\section{Dosimetry and Risk}

Dose estimates for the proposed PET/CT protocols appear in Table 4. The effective dose from the complete PET/CT examination ranged from $8.1 \mathrm{mSv}$ for 4-mo-olds to $13.5 \mathrm{mSv}$ for small adults. These doses represent BERTs ranging from 2.7 to $4.5 \mathrm{y}$. On average, the effective dose from the CT portion is $40 \%$ of the total dose from the PET/CT examination. Figure 6 plots the effective dose across each weight category. For comparison, dosimetry estimates are also plotted for PET/CT examinations with the proposed weight-based injection amount but with a fixed CT acquisition of $120 \mathrm{kVp}$ with 40 and $120 \mathrm{mAs}$ for all weight categories. Likewise, the effective dose from a standard CT chest acquisition, with a fixed adult technique of $200 \mathrm{mAs}$, $120 \mathrm{kVp}$, and 1.375 pitch, is presented to provide a frame of reference. Some centers use higher-dose $\mathrm{CT}$ for attenuation correction and localization. For reference, our proposed PET/ CT protocols would result in a dose that is $50 \%$ for $70-\mathrm{kg}$ patients and $20 \%$ for $7-\mathrm{kg}$ patients of the dose from a PET/CT procedure with a $120-\mathrm{mAs}, 120-\mathrm{kVp}$ CT scan.

Table 4 contains estimates of the LAR of cancer incidence and mortality from the proposed PET/CT protocols. The reported percentages represent highly approximate risk 
FIGURE 2. Graphs show CT noise defined as SD of CT numbers in 4 water phantoms of circumferences ranging from 33 to $79 \mathrm{~cm}$. Errors bars denote SD across 15 circular regions from 3 adjacent slices. Noise vs. tube voltage for fixed tube current of $40 \mathrm{mAs}(\mathrm{A})$ and noise vs. tube current for fixed tube voltage of $120 \mathrm{kVp}(\mathrm{B}) . \mathrm{X}$ in B marks acceptable noise level determined from multiple patient scans of this size patient and used to fix noise for later plots.
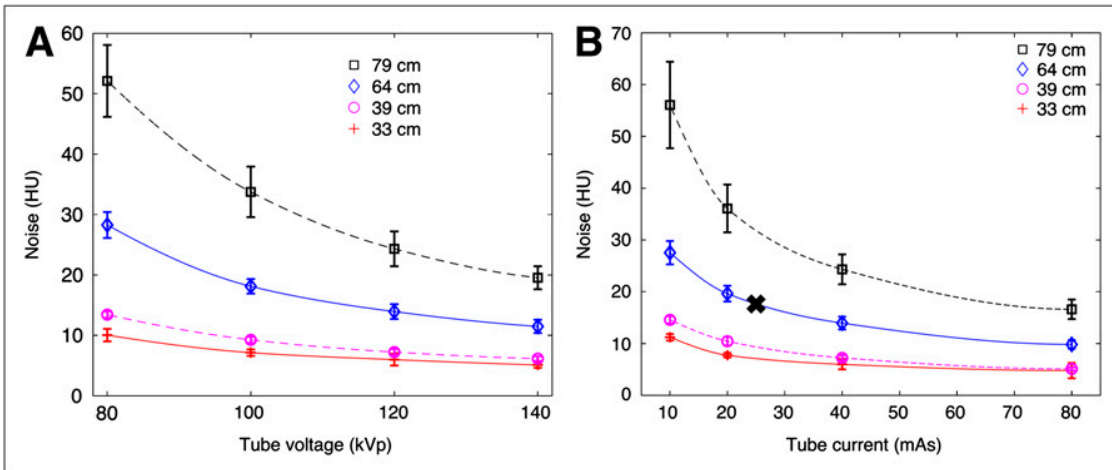

in addition (not multiplicative) to the baseline LAR. For females, the additional risk of acquiring cancer in a lifetime from the PET/CT examination ranged from $0.27 \%$ to $0.38 \%$. Figure 6 plots the female LAR of cancer mortality for the proposed protocols and, for reference, the protocols discussed in the preceding paragraph.

\section{DISCUSSION}

\section{PET Technique}

The proposed weight-based injected activity is based on the widely accepted linear approach used in the United States for pediatric dosing. The proposed linear slope and range represent the median of values used in multiple North American pediatric clinics as presented by Treves et al. (31). The EANM has published suggested guidelines for injected activity of ${ }^{18}$ F-FDG $(30,32)$ (Fig. 1). The guideline's injected activities are based on the work by Jacobs et al. (33) and were proposed in an attempt to normalize the effective dose from radiopharmaceuticals across all ages. A weight-independent effective dose may help simplify the radiation exposure considerations for physicians, but it does

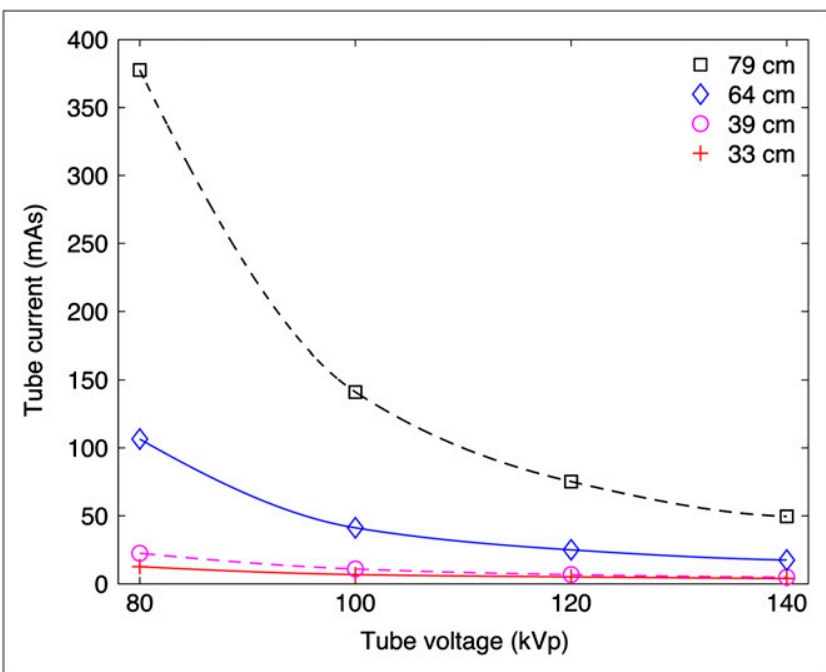

FIGURE 3. Tube current and voltage required to achieve fixed noise level (17.6 Hounsfield units) in 4 water phantoms. not address the more important concern that the same effective dose results in a greater risk of cancer when given to younger patients than when given to older patients. Jacobs et al. acknowledged that a more relevant motivation would be to normalize cancer risk across patients but did not support this approach because their estimates would have resulted in little to no injected activity for the youngest patients. We suggest that the motivation for PET and CT radiation dose should not be guided by normalized cancer risk but rather be driven by the principles of as low as reasonable achievable while maintaining diagnostic utility. In our experience, the proposed injected ${ }^{18} \mathrm{~F}-\mathrm{FDG}$ activity levels provide acceptable image quality for the range of tasks required from the PET images. These suggested activity levels could be reduced on the basis of physician preference or if limited tasks are required, such as the follow-up of known disease.

\section{CT Technique}

For CT, we propose techniques designed for attenuation correction and anatomic correlation of the PET findings. We selected CT techniques based on radiologists' consensus and gradual refinement to desired image quality. We proposed techniques that resulted in different image noise levels, with the trend to require less noise in the smaller patients. The tube voltage requires careful selection to optimize dose efficiency (34). If the tube current remains fixed, as the tube voltage is reduced, the dose to the patient can be reduced substantially (35). Our analysis and the results of the study by Huda et al. (34) show that if the image noise is fixed, as the tube voltage is reduced (and current is increased to maintain the same noise level), the dose to the patient changes only slightly and is a function of patient size. Our results suggest that for the smallest patients we could reduce dose by at best $7 \%$ using 100 $\mathrm{kVp}$ and maintain the same image noise. Conversely, larger patients would have the best dose efficiency at $140 \mathrm{kVp}$ $(\sim 15 \%$ dose savings over $120 \mathrm{kVp}$ ). This trend is in keeping with prior results on soft-tissue lesion contrastto-noise performance in abdominal imaging (36). More rigorous study with a range of anthropomorphic phantoms is required to determine the most dose-efficient tube voltage for each patient. Despite the need for these more 

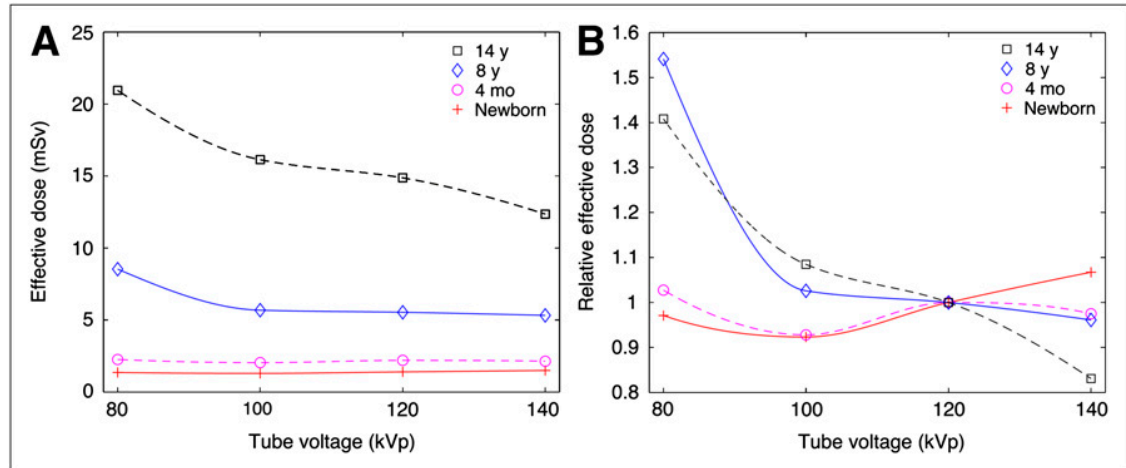

FIGURE 4. Effective dose (A) and relative effective dose normalized to 1 at $120-k V p$ dose $(B)$ vs. tube voltage. Tube current was adjusted at each datum point to achieve fixed noise level (17.6 Hounsfield units) according to Figure 3. Graph offers dose optimization recommendations for each phantom size, ranging from 33 to $79 \mathrm{~cm}$ (patients ranging from newborn to $14-\mathrm{y}$-olds).

rigorous tests, our results suggest the possibility of only modest dose savings at different kilovolt peaks for different patients. Therefore, we have decided to continue to scan all patients with $120 \mathrm{kVp}$ with varying tube currents. At present, for the smallest patients, we are using $10 \mathrm{mAs}$, which is close to the lowest allowable tube current (5 mAs) on the GE Healthcare DVCT system. In the future, if it is determined that images with more noise are acceptable, then the option of reducing the tube voltage may be appropriate to achieve dose savings below the lowest allowable settings for $120 \mathrm{kVp}$. Future work should also analyze the influence of tube voltage on contrast performance, which was not evaluated in this noise analysis.

We recommend using automatic tube current modulation with the proposed CT protocols. We did not discuss automatic exposure control (AEC) in detail because these approaches and parameters are highly vendor-specific. The proposed CT tube currents should be viewed as the maximum current for automatic tube modulation in both the angular and the axial directions. Failure to set a maximum tube current and sole reliance on AEC acquisition parameters are not recommended because these AEC systems
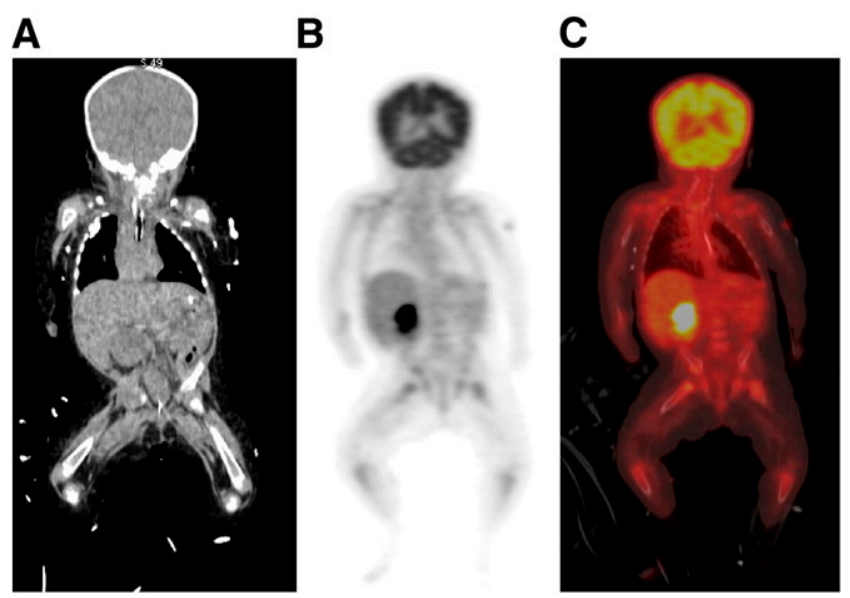

FIGURE 5. Coronal view of CT (A), PET (B), and overlaid $\mathrm{PET} / \mathrm{CT}$ image (C) of 6-kg 4-mo-old. CT was performed with $120 \mathrm{kVp}$ and $10 \mathrm{mAs}$, leading to image with acceptable anatomic detail. PET was performed with $37 \mathrm{MBq}(1 \mathrm{mCi})$ of injected ${ }^{18} \mathrm{~F}-\mathrm{FDG}$ for $3 \mathrm{~min} / \mathrm{bed}$ position. were optimized for the adult patient and may perform suboptimally for small patients.

These anatomic CT techniques were developed to meet our diagnostic preference. Each site should confirm that these techniques meet their clinical needs. Also, the CT techniques were designed on the GE Healthcare DVCT system; the same CT techniques on a system from a different vendor will result in different image quality and patient dose because of variations in detection systems and $\mathrm{X}$-ray tubes.

\section{Dosimetry and Risk}

The proposed PET/CT protocols result in lower estimated effective doses for younger patients, a desired effect in these patients who are more susceptible to radiationinduced adverse effects. The risk estimation indicates that despite the decreased effective dose for the younger patients, the effective cancer risk remains relatively constant across weight categories. The coefficient of variation across categories for the risk of cancer incidence is $18 \%$, and for the risk of cancer mortality it is $12 \%$. This simplifies the discussion of risks from PET/CT examinations across the whole pediatric population. Referencing the highest risk estimates and acknowledging the numerous assumptions inherent in defining risk factors, one could assert that the risk of cancer incidence in a patient's lifetime from 1 of the proposed PET/CT examinations is approximately 38 in 10,000. The corresponding risk of mortality from a cancer due to the proposed PET/CT examinations is approximately 15 in 10,000 .

Dose and risk estimates for CT procedures with a fixed CT technique for each weight category are presented in Figure 6. These results highlight the need to tailor CT procedures for each age or weight category. In a PET/CT examination, if the same CT technique was used for all patients (even if it is a low-dose technique of $40 \mathrm{mAs}$ and $120 \mathrm{kVp}$ ), the additional risk of cancer mortality could be as much as 3 times that from our proposed weight-based technique (10 mAs). Numerous efforts, including the Image Gently campaign, have advocated for dose-conscious pediatric CT procedures (7).

We are not suggesting that these dose and risk estimates be used on a per-patient basis or for detailed risk assess- 
TABLE 4. Estimated Dosimetry and Additional LAR of Cancer for Proposed PET/CT Protocols

\begin{tabular}{|c|c|c|c|c|c|c|c|c|c|c|}
\hline \multicolumn{3}{|c|}{ Patient } & \multicolumn{4}{|c|}{ Approximate effective dosimetry } & \multicolumn{4}{|c|}{ Lifetime attributable risk } \\
\hline & \multirow{2}{*}{$\begin{array}{l}\text { Weight } \\
\text { range } \\
(\mathrm{kg})\end{array}$} & \multirow[b]{2}{*}{$\begin{array}{c}\text { Average } \\
\text { age }\end{array}$} & \multirow[b]{2}{*}{$\begin{array}{l}\text { PET dose } \\
\text { (mSv) }\end{array}$} & \multirow[b]{2}{*}{$\begin{array}{l}\text { CT dose } \\
\text { (mSv) }\end{array}$} & \multirow{2}{*}{$\begin{array}{l}\text { Total effective } \\
\text { PET/CT dose } \\
\text { (mSv) }\end{array}$} & \multirow[b]{2}{*}{ BERT (y) } & \multicolumn{2}{|c|}{ Cancer incidence } & \multicolumn{2}{|c|}{ Cancer mortality } \\
\hline Category & & & & & & & $\begin{array}{c}\text { Females } \\
(\%)\end{array}$ & $\begin{array}{c}\text { Males } \\
(\%)\end{array}$ & $\begin{array}{c}\text { Females } \\
(\%)\end{array}$ & $\begin{array}{c}\text { Males } \\
(\%)\end{array}$ \\
\hline Pink & $6-7.4$ & $4 \mathrm{mo}$ & 5.0 & 3.1 & 8.1 & 2.7 & 0.34 & 0.18 & 0.13 & 0.08 \\
\hline Red & $7.5-9.4$ & $8 \mathrm{mo}$ & 5.1 & 2.9 & 8.0 & 2.7 & 0.33 & 0.18 & 0.12 & 0.08 \\
\hline Purple & $9.5-11.4$ & $15 \mathrm{mo}$ & 5.1 & 4.1 & 9.2 & 3.1 & 0.36 & 0.19 & 0.13 & 0.08 \\
\hline Yellow & $11.5-14.4$ & $2.4 \mathrm{y}$ & 5.5 & 5.2 & 10.7 & 3.6 & 0.38 & 0.21 & 0.15 & 0.09 \\
\hline White & $14.5-18.4$ & $3.8 \mathrm{y}$ & 5.5 & 5.0 & 10.5 & 3.5 & 0.34 & 0.18 & 0.13 & 0.08 \\
\hline Blue & $18.5-22.4$ & 5.9 y & 5.3 & 4.7 & 10.0 & 3.3 & 0.28 & 0.15 & 0.11 & 0.07 \\
\hline Orange & $22.5-31.4$ & $8.5 \mathrm{y}$ & 6.0 & 5.5 & 11.4 & 3.8 & 0.28 & 0.15 & 0.12 & 0.07 \\
\hline Green & $31.5-40.5$ & $10.1 \mathrm{y}$ & 6.6 & 6.3 & 12.8 & 4.3 & 0.29 & 0.16 & 0.12 & 0.08 \\
\hline Black & $40.5-55$ & $12.8 \mathrm{y}$ & 7.5 & 5.6 & 13.1 & 4.4 & 0.27 & 0.15 & 0.12 & 0.08 \\
\hline Small adult & $55-70$ & $15.0 y$ & 7.6 & 5.9 & 13.5 & 4.5 & 0.25 & 0.14 & 0.11 & 0.07 \\
\hline Large adult & $>70$ & $20.0 y$ & 7.0 & 5.9 & 12.9 & 4.3 & 0.19 & 0.11 & 0.09 & 0.06 \\
\hline
\end{tabular}

ment. The intention of these estimates is to provide a frame of reference for comparing radiation dosimetry in PET/CT with other imaging procedures across the population. There remains a debate regarding the use of effective dose $(18,37)$ and the legitimacy of connecting this term with cancer risk as reported in the BEIR VII report (38). The presented dose and risk estimates are approximate. Given the high level of uncertainty in all of the factors, we did not attempt to present error bars on these values. There are several assumptions and simplifications made during the estimation of these factors. The dose estimates are based on simple models of the pediatric population (39) and do not account for the substantial variability of patient morphology, organ sizes, and biokinetics, even for patients of the same age and weight. There are well-appreciated uncertainties in the risk estimates of cancer particularly for the low levels of radiation associated with $\mathrm{PET} / \mathrm{CT}$. These risk numbers should not be viewed as absolute known risks but could be used to compare PET/CT procedures to other radiation procedures.

We evaluated only effective dose and did not address specific organ doses. Organ doses from stand-alone PET procedures have been estimated in the past, and the addition of a low-dose CT acquisition leads to organ doses that are substantially lower than deterministic effect levels $(<200 \mathrm{mSv})$.

PET/CT is often used to monitor response to treatment, requiring multiple examinations. A preliminary report of 248 pediatric PET/CT studies at 1 institution revealed that patients received on average 3.1 PET/CT examinations each (40). At present, the accepted approach is to assume that radiation dose and risk from multiple studies is additive, leading to potentially large cumulative radiation dose and cancer risk.

There is some evidence to support that, along with cancers, radiation exposure leads to increased risk of other diseases. For example, in Nagasaki and Hiroshima fetal exposure to relatively high levels of radiation (120-230 $\mathrm{mSv}$ ) led to a deterministic risk of mental retardation,

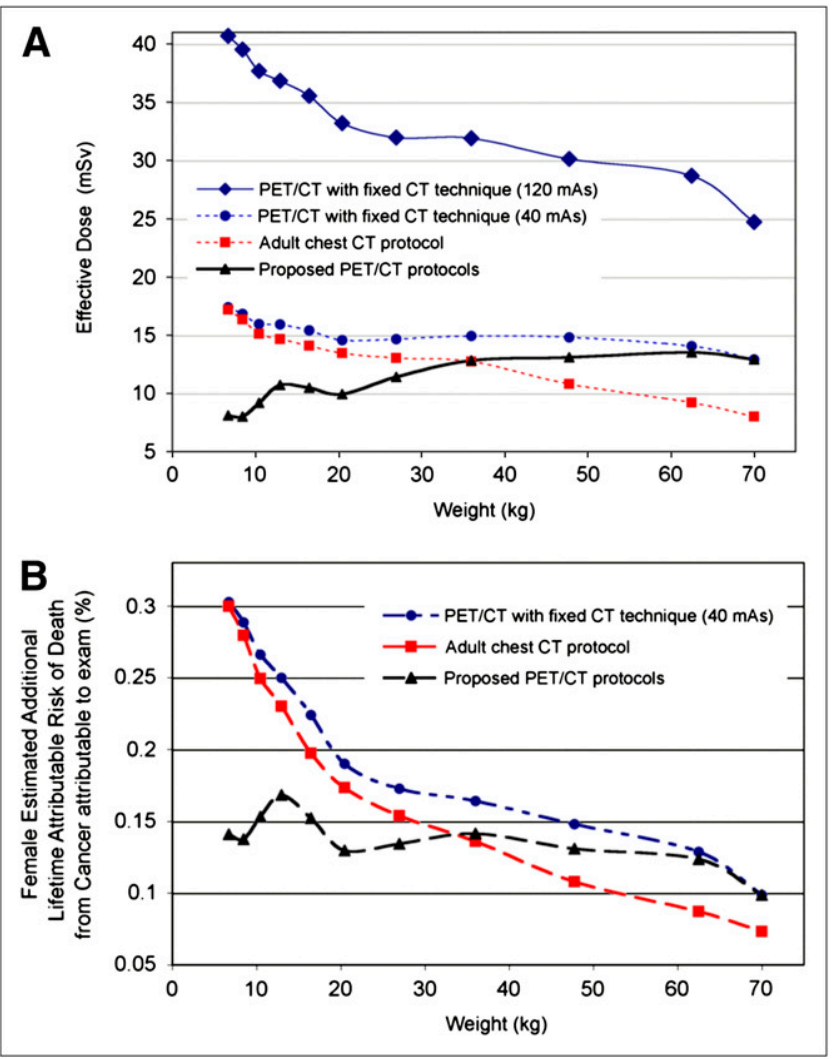

FIGURE 6. (A) Effective dose vs. weight for proposed PET/ CT protocols and CT and PET/CT acquisitions with fixed CT technique. (B) Approximate LAR of cancer mortality for females vs. weight for PET/CT and CT protocols. Symbols are plotted for each color-coded patient category. 
although there is little evidence to support a connection for older children (6). There are also analyses of nuclear industry workers that show a link between radiation exposure and cardiovascular disease (41). The potential risk of additional adverse effects from radiation exposure should be noted, but at present there are not enough data to quantify the increased risk, especially for low levels of radiation and for the pediatric population.

This work presented a discussion of radiation-associated risks of PET/CT. It is beyond the scope of this work to properly present the important benefits of PET/CT and offer a thorough risk or benefit analysis. Lowering radiation exposure will affect the specific tasks associated with PET/ CT, such as detection, localization, characterization, and estimation. A qualitative review of images acquired with the protocols described here yields images that were considered acceptable to allow the localization of anatomic structures with respect to the PET images. This work presents conservative estimates of cancer risk, which are potentially higher than true values. Even with conservative estimates, the risks from PET/CT are generally far outweighed by the benefit of this procedure when used appropriately. Furthermore, pediatric PET/CT is commonly used to diagnose, stage, and monitor already-present cancer, so there is a clearly favorable benefit-to-risk ratio. It is important, however, that the imaging community is aware of the relative amount of additional risk to patients from these imaging procedures.

\section{CONCLUSION}

We proposed whole-body ${ }^{18} \mathrm{~F}-\mathrm{FDG}$ PET/CT protocols for 11 patient weight categories. The use of multiple categories allows for the refinement of acquisition settings to minimize dose while maintaining diagnostic utility. We estimated effective dose and cancer risk from these procedures in an effort to communicate the risk of pediatric PET/CT and evaluate further CT and PET dose-reduction approaches. Future work should seek to determine minimal PET acquisition durations and injected activity and further reductions in CT tube voltage and current for each patient category.

\section{ACKNOWLEDGMENT}

We are grateful to Michael McNitt-Gray for detailed feedback on CT techniques and radiation dose.

\section{REFERENCES}

1. Shulkin BL. PET imaging in pediatric oncology. Pediatr Radiol. 2004;34:199204.

2. Barrington SF, Begent J, Lynch T, et al. Guidelines for the use of PET-CT in children. Nucl Med Commun. 2008;29:418-424.

3. Kaste SC. Issues specific to implementing PET-CT for pediatric oncology: what we have learned along the way. Pediatr Radiol. 2004;34:205-213.

4. Stauss J, Franzius C, Pfluger T, et al. Guidelines for ${ }^{18}$ F-FDG PET and PET-CT imaging in paediatric oncology. Eur J Nucl Med Mol Imaging. 2008;35:15811589 .
5. Committee to Assess Health Risks from Exposure to Low Levels of Ionizing Radiation, Board on Radiation Effects Research, Division on Earth and Life Studies, National Research Council of the National Academies. Health Risks from Exposure to Low Levels of Ionizing Radiation: BEIR VII Phase 2. Washington, D.C.: National Academies Press; 2006.

6. Risk of ionizing radiation exposure to children: a subject review. American Academy of Pediatrics. Committee on Environmental Health. Pediatrics. 1998;101:717-719.

7. Goske MJ, Applegate KE, Boylan J, et al. The 'Image Gently' campaign: increasing CT radiation dose awareness through a national education and awareness program. Pediatr Radiol. 2008;38:265-269.

8. Teras M, Tolvanen T, Johansson JJ, Williams JJ, Knuuti J. Performance of the new generation of whole-body PET/CT scanners: Discovery STE and Discovery VCT. Eur J Nucl Med Mol Imaging. 2007;34:1683-1692.

9. Luten RC, Wears RL, Broselow J, et al. Length-based endotracheal tube and emergency equipment in pediatrics. Ann Emerg Med. 1992;21:900-904.

10. Frush DP, Soden B, Frush KS, Lowry C. Improved pediatric multidetector body CT using a size-based color-coded format. AJR. 2002;178:721-726.

11. Frush DP. Pediatric CT: practical approach to diminish the radiation dose. Pediatr Radiol. 2002;32:714-717.

12. Huda W, Atherton JV, Ware DE, Cumming WA. An approach for the estimation of effective radiation dose at CT in pediatric patients. Radiology. 1997;203:417-422.

13. Fahey FH, Palmer MR, Strauss KJ, Zimmerman RE, Badawi RD, Treves ST. Dosimetry and adequacy of CT-based attenuation correction for pediatric PET: phantom study. Radiology. 2007;243:96-104.

14. Gelfand MJ, Lemen LC. PET/CT and SPECT/CT dosimetry in children: the challenge to the pediatric imager. Semin Nucl Med. 2007;37:391-398.

15. Hurwitz LM, Reiman RE, Yoshizumi TT, et al. Radiation dose from contemporary cardiothoracic multidetector CT protocols with an anthropomorphic female phantom: implications for cancer induction. Radiology. 2007;245:742-750.

16. Shrimpton PC, Jones DG. Normalised organ doses for $\mathrm{x}$ ray computed tomography calculated using Monte Carlo techniques and a mathematical anthropomorphic phantom. Radiat Prot Dosimetry. 1993;49:241-243.

17. Valentin J. Radiation dose to patients from radiopharmaceuticals (addendum 2 to ICRP publication 53) ICRP publication 80 approved by the Commission in September 1997. Ann ICRP. 1998;28:1.

18. McCollough $\mathrm{CH}$, Schueler BA. Calculation of effective dose. Med Phys. 2000;27:828-837.

19. International Commission on Radiological Protection (ICRP). 1990 Recommendations of the International Commission on Radiological Protection. Publication 60. Oxford, England: Pergamon Press; 1991.

20. Mettler FAJ, Thomadsen BR, Bhargavan M, et al. Medical radiation exposure in the U.S. in 2006: preliminary results. Health Phys. 2008;95:502-507.

21. Kuczmarski RJ, Ogden CL, Grummer-Strawn LM, et al. CDC growth charts: United States. Adv Data. 2000;314:1-27.

22. Niven E, Nahmias C. Absorbed dose to very low birth weight infants from ${ }^{18} \mathrm{~F}$ fluorodeoxyglucose. Health Phys. 2003;84:307-316.

23. ImPACT Group. CT dosimetry tool. London, England: ImPACT, St. George's Healthcare NHS Trust, 2007.

24. Khursheed A, Hillier MC, Shrimpton PC, Wall BF. Influence of patient age on normalized effective doses calculated for CT examinations. Br J Radiol. 2002; 75:819-830.

25. Huda W, Ogden KM. Computing effective doses to pediatric patients undergoing body CT examinations. Pediatr Radiol. 2008;38:415-423.

26. Cardis E, Vrijheid M, Blettner M, et al. The 15-country collaborative study of cancer risk among radiation workers in the nuclear industry: estimates of radiation-related cancer risks. Radiat Res. 2007;167:396-416.

27. Nussbaum RH. The linear no-threshold dose-effect relation: is it relevant to radiation protection regulation? Med Phys. 1998;25:291-299.

28. Brenner D, Sachs R. Estimating radiation-induced cancer risks at very low doses: rationale for using a linear no-threshold approach. Radiat Environ Biophys. 2006;44:253-256.

29. International Commission on Radiological Protection (ICRP). ICRP 99: lowdose extrapolation of radiation-related cancer risk. Ann ICRP. 2005;35:1-142.

30. Lassmann M, Biassoni L, Monsieurs M, Franzius C. The new EANM paediatric dosage card: additional notes with respect to F-18. Eur J Nucl Med Mol Imaging. 2008 ;

31. Treves ST, Davis RT, Fahey FH. Administered radiopharmaceutical doses in children: a survey of 13 pediatric hospitals in North America. J Nucl Med. 2008;49:1024-1027.

32. Lassmann M, Biassoni L, Monsieurs M, Franzius C, Jacobs F. The new EANM paediatric dosage card. Eur J Nucl Med Mol Imaging. 2007;34:796-798.

33. Jacobs F, Thierens H, Piepsz A, et al. Optimised tracer-dependent dosage cards to obtain weight-independent effective doses. Eur J Nucl Med Mol Imaging. 2005;32:581-588. 
34. Huda W, Ogden KM, Khorasani MR. Effect of dose metrics and radiation risk models when optimizing CT x-ray tube voltage. Phys Med Biol. 2008;53:47194732 .

35. Gkanatsios NA, Huda W, Peters KR. Effect of radiographic techniques ( $\mathrm{kVp}$ and mAs) on image quality and patient doses in digital subtraction angiography. Med Phys. 2002;29:1643-1650.

36. Huda W, Ogden KM. Optimizing abdominal CT dose and image quality with respect to x-ray tube voltage. Proc SPIE. 2004;5368:499-507.

37. Shrimpton PC, Wall BF, Yoshizumi TT, Hurwitz LM, Goodman PC. Effective dose and dose-length product in CT. Radiology. 2009;250:604-605.
38. Brenner DJ, Hall EJ. Computed tomography: an increasing source of radiation exposure. N Engl J Med. 2007;357:2277-2284.

39. Cristy M, Eckerman KF. Specific Absorbed Fractions of Energy at Various Ages from Internal Photon Sources. Oak Ridge, TN: Oak Ridge National Laboratory; 1987.

40. Chawla S, Federman N, Nagata K, et al. Estimated cumulative radiation dose from PET/CT in pediatric patients with malignancies: a 5-year retrospective review [abstract]. Pediatr Radiol. 2008;38(suppl):S339.

41. McGeoghegan D, Binks K, Gillies M, Jones S, Whaley S. The non-cancer mortality experience of male workers at British Nuclear Fuels plc, 1946-2005. Int J Epidemiol. 2008;37:506-518.

\section{Errata}

In the article "11 C-Dihydrotetrabenazine PET of the Pancreas in Subjects with Long-Standing Type 1 Diabetes and in Healthy Controls," by Goland et al. (J Nucl Med. 2009;50:382-389), the name of one of the acknowledged individuals, Arthur Mikhno, was misspelled. The authors regret the error.

A methodologic error appeared in the article "Highly Efficient In Vivo Agonist-Induced Internalization of sst ${ }_{2}$ Receptors in Somatostatin Target Tissues," by Waser et al. ( Nucl Med. 2009;50:936-941). In the second section of the "Materials and Methods," the sentence "Both primary antibodies were applied in a 1:1,000 dilution." should have read "Both R2-88 and SS-800 were applied in a 1:1,000 dilution, whereas a 1:100 dilution was necessary for UMB-1." The authors regret the error.

In the article "Influence of Trigger PSA and PSA Kinetics on ${ }^{11} \mathrm{C}$-Choline PET/CT Detection Rate in Patients with Biochemical Relapse After Radical Prostatectomy," by Castellucci et al. (J Nucl Med. 2009;50:1394-1400), the legend of Figure 5 describes the illustrated patient as being $1 \mathrm{y}$ old instead of the correct age, $71 \mathrm{y}$. We regret the error. 